\title{
ANÁLISE DAS PERSPECTIVAS DE APLICAÇÕES DAS LIGAS COM MEMÓRIA DE FORMA POR MEIO DE LEVANTAMENTO DE PATENTES*
}

\section{Resumo}

Murillo Romero da Silva ${ }^{1}$ Cláudio Shyinti Kiminami² Daniel Rodrigo Leiva ${ }^{3}$ Piter Gargarella ${ }^{3}$ Claudemiro Bolfarini²

Ligas com memória de forma (LMFs) são ligas metálicas capazes de recuperar o seu formato e tamanho original após deformação plástica quando aquecidas acima de uma temperatura crítica. Diversos casos de possíveis aplicações paras as LMF têm sido justificados no campo científico, com alguns poucos casos identificados para aplicação em massa, existindo uma ausência na literatura de uma análise crítica da aplicabilidade das mesmas que leve em consideração aspectos industriais e de mercado. Dessa forma, se faz necessário um estudo mais aprofundado que correlacione as ideias científicas geradas com a produção industrial e com a comercialização de LMFs. Nesse contexto, o trabalho visa através de levantamento e análise de patentes e artigos científicos, assim como por contatos industriais, estabelecer uma relação entre o desenvolvimento científico e o comercial das LMFs. Mais de 11 mil artigos e mais de 7 mil patentes foram produzidos nos últimos 16 anos, sendo observado uma tendência crescente da criação de conhecimento de LMF, o qual já vem sendo aplicado na indústria, justificando assim os esforços observados no campo científico. A área biomédica se destaca como a mais bem estabelecida no campo das LMF, com destaque para a utilização das ligas a base de NiTi.

Palavras-chave: Ligas com memória de forma; Aplicabilidade; Patentes; Indústria.

\section{ANALYSIS OF SHAPE MEMORY ALLOYS APPLICATION PERSPECTIVE BY SURVEY AND ANALYSIS OF PATENTS}

\section{Abstract}

Shape Memory Alloys (SMA) are metallic alloys capable of recovering their original shape and size after plastic deformation when heated above a critical temperature. Many potentials applications have been justified in scientific field, with just few of them being for mass application. There is an absence in the literature of an analysis about applicability of SMAs, which takes into account industrial and market aspects. Thus, a further study is necessary in order to correlate the scientific ideas generated with the industrial production and commercialization of the SMAs. In this context, the present work aims through survey and analysis of patents and scientific papers, as well as through industrial contacts, stablish a relationship between the scientific and the commercial development of SMAs. More than 11 thousand articles and more than 7 thousand patents were produced in the last 16 years. It was observed a growing tendency of knowledge creation about SMAs, which is already being applied in industrial field, justifying the efforts observed in scientific field. The biomedical area stands out as the most well-stablished in the production of SMA, and the NiTi SMA stands out as the SMA most used on the industry overall.

Keywords: Shape Memory Alloys; Applicability; Patents; Industry.

1 Engenheiro de Materiais. Estudante de Mestrado no Programa de Pós-Graduação em Ciência e Engenharia de Materiais, Universidade Federal de São Carlos (UFSCar), São Carlos, SP, Brasil.

2 Engenheiro de Materiais. Professor Associado do Departamento de Engenharia de Materiais, UFSCar, São Carlos, SP, Brasil.

3 Engenheiro de Materiais. Professor Adjunto do Departamento de Engenharia de Materiais, UFSCar, São Carlos, SP, Brasil. 


\section{INTRODUÇÃO}

Ligas com memória (LMF) de forma caracterizam-se como ligas que apresentam o efeito de memória de forma (EMF), ou seja, capacidade de retornar a seu formato original após deformação plástica quando aquecidas acima de uma temperatura crítica [1][2][3][4]. Essas ligas também apresentam outras características como pseudoelasticidade e alta capacidade de amortecimento [2], as quais possibilitam uma vasta gama de aplicações para essa classe de materiais.

Entre as ligas com memória de forma se destacam aquelas à base de $\mathrm{Ti}$ e $\mathrm{Ni}$, que apresentam ótimas propriedades mecânicas e excelente biocompatibilidade, e as ligas a base de $\mathrm{Cu}$, que não apresentam propriedades mecânicas elevadas, mas apresentam custo de produção reduzido [3][5]. Essas ligas apresentam propriedades variadas, as quais culminam com um aumento no potencial para aplicações comerciais [4].

Nos últimos anos, as ligas com memória de forma vêm recebendo uma importância maior no campo científico e diversas aplicações têm sido apresentadas, sendo essas em áreas diversas que abrangem tanto biomateriais, como sensores e atuadores [6]. Entretanto, apenas justificativas no campo científico têm sido propostas, com poucas aplicações sendo realmente observadas no meio industrial. Dessa forma, na literatura, não é observado dados que mostrem de forma esclarecedora se as informações obtidas cientificamente estão sendo exploradas no âmbito comercial - industrial, ou seja, para a produção de produtos.

Nesse contexto, o presente trabalho tem como objetivo realizar uma análise da aplicabilidade das LMF utilizando de uma análise das patentes registradas com o tema de LMF, observando as empresas que mais registraram patentes nos últimos 20 anos, visando correlacionar a produção industrial dessas empresas com o conhecimento cientifico gerado nessa área. A partir desse estudo, o trabalho visa esclarecer quais áreas estão mais bem consolidadas na produção de LMF, assim como quais produtos estão sendo produzidos e quais ligas são utilizadas para a produção de produtos com elementos de memória. A análise a respeito dessas ligas tende a gerar um direcionamento da pesquisa em função da demanda de mercado e do potencial de processamento e comercialização do material, levando a um melhor entendimento no que tange a correlação pesquisa, produção e mercado, e assim, possibilitando uma melhor compreensão do potencial de aplicação dessas ligas, tanto no contexto atual, quanto para o contexto futuro.

\section{MATERIAIS E MÉTODOS}

Foi realizado pesquisa de patentes utilizando o banco de dados fornecido pela Web of Science ${ }^{S M}$ (http://apps.webofknowledge.com). Para a pesquisa de patentes utilizouse a base de dados Derwent Innovations Index ${ }^{S M}$, sendo a pesquisa realizada no modo "pesquisa avançada" utilizando a palavra-chave "Shape Memory Alloy". A Derwent Innovations Index ${ }^{S M}$ é uma base de dados da Thomson Reuters. A base de dados é atualizada semanalmente e contém mais de 16 milhões de invenções práticas, desde 1963 até os dias de hoje. As informações de patente são coletadas com 41 autoridades emissoras de patente em todo o mundo, inclusive no Brasil [7].

$\mathrm{Na}$ pesquisa realizada efetuou-se um levantamento de patentes registradas relativas ao período de 1963 até o ano de 2016, de forma a observar a evolução do processo de patenteamento, e pesquisa, no campo de ligas com memória de forma durante este período. 
As patentes registradas de 1963 até 1995 já não se encontram protegidas pelo sistema de patentes visto que já foi ultrapassado o período de vigência da proteção relativa a uma patente, o qual corresponde a 20 anos. Dessa forma, um estudo mais aprofundado a respeito das patentes registrados a partir do ano 2000 foi realizado desconsiderando em parte as patentes obtidas nos anos de 2014 e 2016, uma vez que o processo de registros de patentes pode demorar 18 meses para ser aprovado, o que resultaria em incoerências no percentual de patentes observadas durante esse período [8].

Em seguida, através dos dados de registros de patentes obtidos foi realizado um levantamento dos principais depositantes de patentes, de forma a procurar os responsáveis pelo interesse na proteção das informações tecnológicas obtidas no campo de ligas com memórias de forma. Os depositantes em geral correspondem a grandes companhias, as quais possuem o capital necessário para suprir o preço requerido para o patenteamento de uma ideia científica. Esse interesse está diretamente atrelado a questões produtivas, comerciais e industriais.

A partir do levantamento dos principais depositantes, foi realizado um ranqueamento das cincos empresas com maior número de registro de patentes. Elas tiveram suas patentes analisadas, assim como seus respectivos websites e suas informações contidas na internet, de forma a procurar estabelecer uma relação entre as ideias patenteadas pela empresa e a produção da mesma.

Após análise das patentes, foi realizado um levantamento de artigos publicados no período de 1945 até 2016 com a palavra-chave "Shape Memory Alloy", utilizando novamente a base de dados da Web of Science ${ }^{\mathrm{TM}}$ de forma a se observar a evolução da publicação de artigos relacionados a LMF no decorrer dos anos, e através desses dados comparar com aqueles obtidos para as patentes registradas com o tema LMF.

\section{RESULTADOS E DISCUSSÃO}

\subsection{Análise de patentes e artigos}

Através da pesquisa de patentes efetuada encontrou-se os seguintes dados que podem ser visualizados nas Figuras 1:

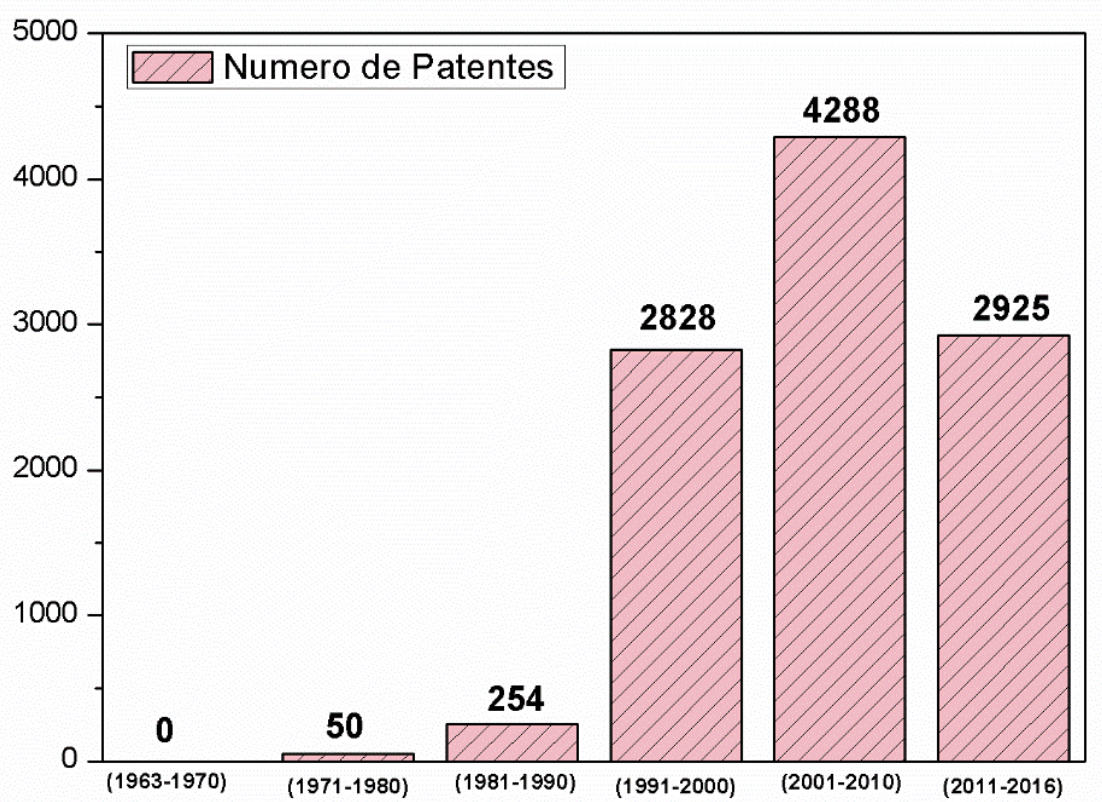

Figura 1. Número de patentes registradas no período de 1963 a 2016 com o tema de LMF. 
Observa-se através da Figura 1 que o número de patentes registradas nos últimos anos, a partir da década de 60 , aumentou de forma abrupta, visto que no período de 10 anos entre 1971 e 1980 observou-se o número de apenas 50 patentes registradas, ao passo que no decênio de 2001 a 2010 esse número ultrapassou a quantidade de 4200 patentes registradas. Apesar do período de 2010 a 2016 corresponder a apenas 6 anos, e levando-se em consideração que algumas patentes geradas no período de 2014 a 2016 ainda estarem em processo de aprovação, percebe-se que o valor de 2925 patentes registradas se encontra dentro da média de crescimento observada, o que caracteriza que o processo de registros de patentes com o assunto envolvendo LMF está em processo contínuo de crescimento.

O resultado obtido para o levantamento de artigos publicados no período de 1945 a 2016 pode ser visualizado na Figura 2:

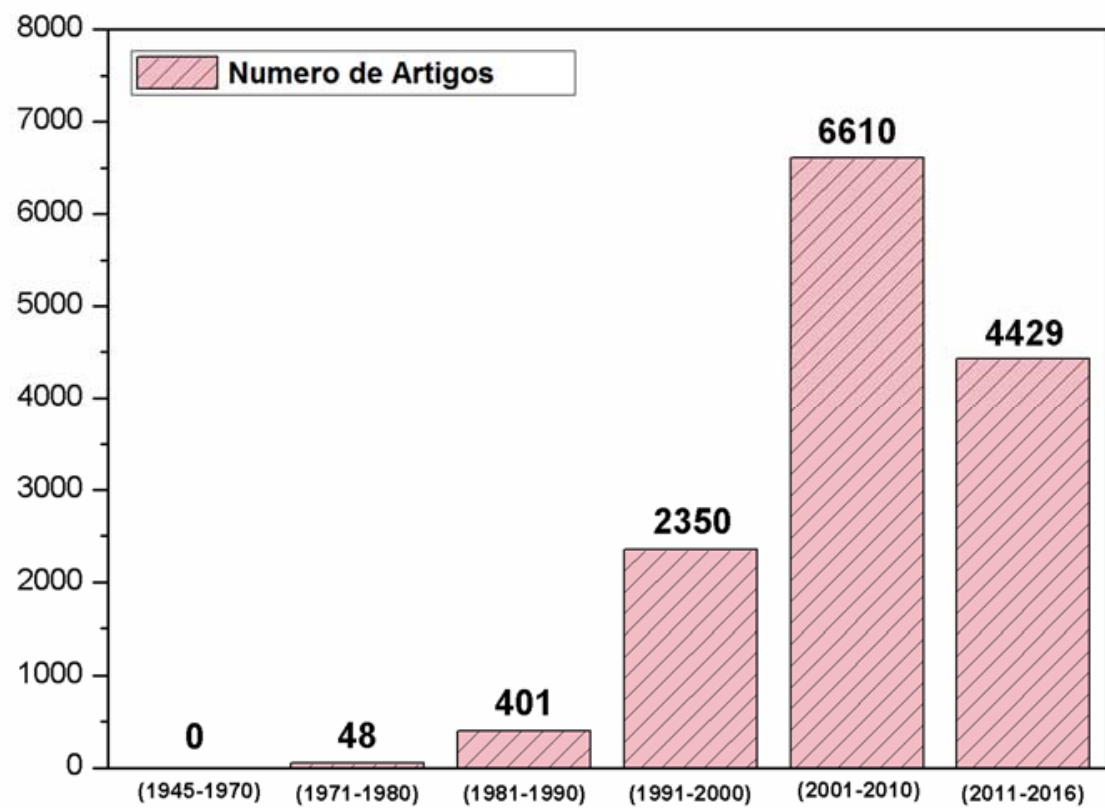

Figura 2. Número de artigos publicados no período de 1945 a 2016 com o tema de LMF.

O comportamento dos artigos publicados de 1945 até 2016 segue o mesmo padrão observado acima para as patentes registradas, ocorrendo um crescimento abrupto a partir da década de 80. Até o período de 1980, 20 anos após a descoberta das LMFs, a produção de artigos científicos relacionados a área foi ínfima, computando o valor de apenas 48 artigos. Um grande crescimento pode ser observado no final do século 20, onde 2350 artigos foram publicados de 1991 a 2000, e no começo do século 21, de 2001 a 2010, onde foi observado quase o triplo de artigos publicados comparado com a década anterior. No período de 2011 a 2016, o qual corresponde a apenas um pouco mais de 5 anos, o valor já registrado de artigos publicados mostra, que assim como as patentes registradas, o processo de publicação de artigos relacionados ao tema LMF está em crescimento contínuo.

Os dados obtidos relativos as patentes registradas e dos artigos publicados durante o período analisado estão de acordo com alguns dados encontrados na literatura. Mohd Jani et al. [6] utilizou a base de dados SCOPUS e USPTO com a palavra-chave "Shape Memory Alloy" e obteve resultados similares aos do presente trabalho. A SCOPUS corresponde a um banco de dados de resumos e citações de artigos para jornais/revistas acadêmicas, e patentes, que abrange cerca de 19,5 mil títulos de mais de 5 mil editoras internacionais. A SCOPUS pertence a editora Elsevier e abrange os cinco principais escritórios de patentes no mundo: WIPO, USPTO, European Patent 
Office, UK Patent Office e Japonese Patent Office. A USPTO (United Stades Patent and Trademark Office) corresponde a um escritório norte-americano de patentes, 0 qual regula a concessão de patentes nos EUA, assim como o registro de marcas [9]. Além de mostrar o crescimento observado no número de patentes com a palavrachave "Shape Memory Alloy" Mohd Jani et al. [6] também mostrou uma projeção para o período de 2010 a 2019, no qual mais de 25 mil patentes são esperadas, correspondendo a um incremento significativo na produção de conhecimento cientifico de LMF nos próximos anos. Mohd Jani et al. [6] também apresentou a evolução do número de publicações de artigos relacionadas com LMF, observando-se um crescimento no número de publicações, mostrando também uma projeção elevada de cerca de 30 mil artigos para o período de 2010 a 2019.

O crescimento observado e projetado para o futuro, tanto para patentes registradas como para artigos publicados, nos mostra que existe um interesse crescente no desenvolvimento de conhecimento cientifico para com o tema LMF. Esse crescente interesse possivelmente está atrelado a indústria no que tange a possível diminuição de custos agregados ao produto, assim como na melhoria da tecnologia empregada no processo ou no produto através da utilização das LMF.

Em uma outra análise de Mohd Jani et al. [6] temos que entre as áreas que mais registraram patentes e publicaram artigos relacionados ao tema LM observa-se que a área biomédica se apresenta como aquela que mais registrou patentes no período analisado, com um percentual de $61 \%$, sendo seguido pelas áreas automobilística, aeroespacial e robótica.

Apesar de existir um elevado número de artigos publicados, assim como de patentes registradas, observa-se ainda uma ausência de dados que permita concluir se as ideias patenteadas estão sendo utilizadas em produtos. Uma forma utilizada para conectar os dados obtidos de patentes registradas com a produção industrial se encontra no depositante das patentes, o qual corresponde ao responsável pelo pagamento das despesas referentes ao processo de patenteamento. Dessa forma, empresas interessadas na proteção de ideias cientificas adquiridas realizam 0 pagamento das despesas de patenteamento, e tem assim seu nome constado no arquivo de patentes, podendo este ser visualizado.

Utilizando dos nomes de depositantes presentes nas patentes obtidas em uma nova pesquisa realizada no trabalho, a qual abrange somente as patentes registradas nos últimos 16 anos, realizou-se um levantamento com os depositantes que apresentam o maior número de depósitos de patentes, o que pode ser visualizados na Tabela 1. Através do rankeamento das companhias depositantes podemos inferir que estas são as principais interessadas no desenvolvimento de novos produtos relacionadas com LMF, e dessa forma são um ponto de partida para uma análise de mercado.

$\mathrm{Na}$ Tabela 1 pode se observar as 25 empresas que mais realizaram o depósito para o patenteamento dos documentos de patentes relacionados a LMFs durante o período de 2000 a 2016. As áreas de atuação das respectivas empresas também podem ser visualizadas na Tabela 1. A pesquisa realizada obteve uma quantidade de 7468 patentes registradas no período, e conforme pode ser visualizado na Tabela 1, as 25 maiores depositantes registraram ao todo 1659 patentes, o que corresponde a $22,1 \%$ do total registrado no período. 
Tabela 1 - Empresas depositantes de patentes, área de atuação e respectivo número de patentes.

\begin{tabular}{|c|c|c|}
\hline Empresa & No $^{\circ}$ de Patentes & Área de Atuação \\
\hline GM Global Technology Operations Inc. & 364 & Automobilística \\
\hline Konica Minolta Opto KK & 141 & Eletroeletrônicos \\
\hline Boston Sci Scimed Inc. & 99 & Biomédica \\
\hline Cook Medical Technologies LLC & 84 & Biomédica \\
\hline Matsushita Denki Sangyo KK & 84 & Eletroeletrônicos \\
\hline Boeing CO & 74 & Aeronáutica \\
\hline Tokin Corp & 69 & Dispositivos Eletrônicos \\
\hline Medtronic Inc. & 67 & Biomédica \\
\hline Olympus Optical LTD & 61 & Eletroeletrônicos \\
\hline Johnson NL & 55 & Automobilística \\
\hline Hyundai Motor CO LTD & 54 & Automobilística \\
\hline Univ. Shangai Jiatong & 53 & Acadêmica \\
\hline Samsung Eletronic CO LT & 53 & Eletroeletrônicos \\
\hline Dokuritsu Gyosei Hojin Sangyo Gijutsu So & 51 & Biomédica \\
\hline Sony Corp. & 46 & Eletroeletrônicos \\
\hline Harbin Inst. Technology & 40 & Acadêmica \\
\hline Univ. Beijing Aeronautisc \& Austronautics & 37 & Acadêmica \\
\hline Browne AL & 36 & Automobilística \\
\hline Siemens AG & 34 & Eletroeletrônicos \\
\hline Warsaw Orthopedic Inc. & 33 & Biomédica \\
\hline Univ. Dalian Technology & 32 & Acadêmica \\
\hline Hon Hai Precision Ind. CO Ltd. & 27 & Eletroeletrônicos \\
\hline Zhenjiang Yinuowei Memory Alloy LTD & 31 & Eletroeletrônicos \\
\hline Advanced Cardiovascular System & 32 & Biomédica \\
\hline Dynalloy INC & 30 & Eletroeletrônicos \\
\hline & & \\
\hline & & \\
\hline
\end{tabular}

Entre os depositantes presentes na Tabela 1 podemos perceber que existe uma predominância de empresas relacionadas a área de eletroeletrônicos. Entre as cinco maiores depositantes podemos visualizar a presença de duas empresas relacionadas com a área biomédica, a qual vem sendo apontada como a área de maior produção de registros de patentes com o tema de liga com memória de forma [6].

As cinco primeiras empresas na Tabela 1 foram observadas com uma maior atenção de forma a procurar indícios a respeito da utilização da ideia científica no processo industrial, ou seja, na transformação do conhecimento científico adquirido em produto comercializável.

\subsection{General Motors Corporation}

A GM na pesquisa realizada no trabalho apresentou-se como a líder dos depositantes de patentes com o termo LMF com o total de 364 patentes, o que corresponde a quase o triplo do número de patentes publicados pela segunda colocada Konica Minotta Opto.

A análise dessas patentes registradas pela GM nos mostra que existem estudos com LMF para criação e melhoria de atuadores, os quais representam cerca de $80 \%$ das patentes registradas. Os atuadores encontrados nas patentes registradas pela GM possuem diversas funções, e trabalham basicamente utilizando as propriedades intrínsecas das LMF: EMF e pseudoelasticidade. A variação de temperatura ou tensão promove uma variação na forma do dispositivo, mudança essa que coordena o funcionamento do atuador.

Os atuadores de LFM vem sendo amplamente estudados no campo automobilístico uma vez que substituem os atuais atuadores elétricos, os quais são maiores em 
tamanho e possuem uma geometria mais complexa. Dessa forma, além de se obter um barateamento no custo de produção para os atuadores, uma redução de peso também seria observada, indo de encontro com os objetivos da indústria automobilística.

Foi utilizado o endereço virtual da GM (http://www.gm.com) para verificar se a empresa já estaria utilizando em alguns de seus produtos as ideias oriundas das patentes registradas, de forma a encontrar uma conexão entre pesquisa e produção de LMF. O único produto de LMF encontrado foi no veículo Corvette 2014, onde utilizase um atuador de liga com memória de forma no lugar de um motorizado [10]. $O$ atuador abre e fecha a abertura de ventilação, que é alocada na parte traseira de forma escondida, e que libera o ar a partir do porta malas. O fio de atuador de LMF utilizado no Corvette 2014 é $0.5 \mathrm{~kg}$ mais leve que o utilizado no modelo anterior [10]. Considerando que existem mais de 200 partes móveis motorizadas que podem ser substituídas por smart materials como o mostrado anteriormente em um veículo, uma grande redução de massa pode ser obtida no futuro.

\subsection{Konica Minolta Inc.}

A empresa apresenta um total de 141 patentes registradas relacionadas com o tema LMF, ocupando a segunda posição na Tabela 1. Assim como nas patentes analisadas da GM, discutidas acima, as patentes registradas pela Konica Minolta são em sua grande maioria voltadas para o desenvolvimento de atuadores. Os atuadores descritos nas patentes da Konica Minolta exercem diversas funções em aparelhos de geração de imagem, destacando-se o dispositivo de condução em uma câmera fotográfica de celular, onde o elemento de memória de forma é o gerador da força motriz para o movimento das unidades de lente ou de espelhos. Nesse caso o tamanho do dispositivo seria menor que os que vem sendo utilizado o que salvaria espaço no aparelho além de reduzir o custo agregado do mesmo. A empresa também registrou patentes de atuadores que possam operar em projetores de imagens, em dispositivos de condução de endoscópios e em motores para o mecanismo de focagem e zoom.

Foi utilizado o website da empresa Konica Minolta Opto (http://www.konicaminolta.com/), após a análise das patentes registradas, para verificar se existe uma conexão entre as ideias presentes nas patentes com a produção da empresa. Foi realizada a pesquisa por produtos com o termo "Shape Memory Alloy", "SMA" e "Nitinol" e nenhum produto foi obtido pela pesquisa. É possível que a empresa não produza produtos com liga de memória de forma devido a venda do departamento de fotografia digital para a Sony em 2006, departamento esse que apresentava a maior quantidade de patentes relacionadas com liga com memória de forma da Konica Minolta.

\subsection{Boston Scimed}

A empresa se encontra na terceira posição da Tabela 1 com 99 patentes registradas com o termo ligas com memória de forma. Entretanto, se tratando de uma área médica as patentes exploram uma área diferente daquela observada nos dois casos anteriores. As patentes registradas pela empresa são em geral para o desenvolvimento de produtos relacionados a cateteres, stents e grampos cirúrgicos, utilizando principalmente do efeito de super-elasticidade apresentado pelas LMFs. 
Observa-se nas patentes registradas que ocorre uma predominância de ligas a base de $\mathrm{NiTi}$, uma vez que apresentam melhor biocompatibilidade e melhores propriedades mecânicas, as quais são propriedades requeridas e fundamentais para a aplicação médica.

Após análise das patentes produzidas pela Boston Scimed, foi utilizado o website da empresa (http://www.bostonscientific.com/en-US/home.html) para verificar se existe uma conexão entre as ideias presentes nas patentes e da produção da empresa. Foi realizada a pesquisa por produtos com o termo "Shape Memory Alloy" ou "SMA" e apenas 2 resultados foram obtidos. Entretanto, para a pesquisa realizada com o termo "Nitinol" foi encontrado o resultado com 63 produtos.

Dessa forma foi possível observar que a empresa Boston Scimed já utiliza amplamente em sua produção as ideias oriundas das patentes registradas com 0 termo de LMF, sendo observado no website da empresa diversos produtos como fio guias, stents e grampos cirúrgicos, os quais estão de acordo com as ideias registradas nas patentes observadas.

\subsection{Cook Medical}

A empresa apresentou 84 patentes registradas com o termo LMF na pesquisa realizada no trabalho, ocupando a $4^{a}$ posição na Tabela 1. As ideias apresentadas nas patentes observadas são voltadas para a produção de stents, fios-guia e cateteres. Assim como para a Boston Scimed não se observou a presença elevada de atuadores nas patentes analisadas da Cook Medical, e também pode se observar uma preferência pelo uso de ligas de NiTi uma vez que essas apresentam uma maior biocompatibilidade, propriedade essa necessária uma vez que os produtos gerados pelas patentes irão operar em contato com o corpo humano.

Foi utilizado o website da empresa Cook Medical (https://www.cookmedical.com/), onde se realizou a pesquisa por produtos com o termo "Shape Memory Alloy" ou "SMA" e apenas 2 resultados foram obtidos. Entretanto, assim como realizado para a Boston Scimed, foi também realizado pesquisa com o termo "Nitinol", e obteve-se 80 resultados, dos quais 39 correspondem a produtos da empresa. Dessa forma, foi verificado que a Cook Medical já utiliza amplamente em sua empresa e em sua produção as ideias patenteadas relativas a LMF.

Assim, pode ser notado que as empresas da área biomédica já dominam bem algumas técnicas de produção e também se apresentam consolidadas na comercialização dos produtos que utilizam LMF.

\subsection{Panasonic Corporation (Matshushita Denki Sangyo)}

A empresa apresentou 84 patentes registradas com o tema LMF, sendo ranqueada na quinta posição da Tabela 1 . As ideias presentes nas patentes registradas pela empresa estão atreladas principalmente a produção de atuadores, visto que se trata de uma empresa de eletrônicos e a utilização desses atuadores de memória de forma tendem a diminuir o custo e o peso dos itens produzidos pela empresa.

Seguida a análise das patentes registradas pela Panasonic Corporation, foi realizado uma pesquisa no website da empresa (http://panasonic.net/), onde não foi encontrado nenhum produto relacionado a LMF, sendo realizado pesquisas com "Nitinol", "Shape Memory Alloy" e "SMA". 


\section{CONCLUSÃO}

No campo da pesquisa existe um claro interesse no estudo e no desenvolvimento do tema LFM. É observado um crescimento nítido tanto no número de patentes registradas como no número de artigos publicados relacionados ao tema de LMF, e as projeções mostram que esse crescimento tende a continuar, mostrando nitidamente o grande interesse que vem sendo depositado no ramo de pesquisa dentre dessa área analisada.

Esse estudo crescente de LMF é justificado uma vez que as ideias cientificas, observadas em artigos e patentes, encontram inseridas na produção industrial, sendo observada na forma de produto em três das cinco empresas analisadas.

Dentre as ligas com memória de forma analisadas, observa-se um predomínio massivo das ligas TiNi nas patentes registradas e nos produtos produzidos pelas empresas analisadas. Isso no leva a concluir que embora o maior custo de processamento, as melhores propriedades dessa classe de liga são preponderantes no fator de seleção para a produção de produtos com memória de forma.

A indústria biomédica é aquela que se encontra mais bem estabelecida no que tange a implementação das ideias cientificas em sua produção. Isso vem de encontro com estudos que mostram que a área biomédica representa aquela de maior produção de material cientifico relacionado a LMF. Os produtos produzidos com LMF pelas empresas biomédicas são principalmente cateteres, fios-guia, stents e grampos cirúrgicos, sendo a liga TiNi a liga mais utilizada principalmente devido a sua excelente biocompatibilidade.

A área automobilística apresenta um interesse elevado na produção de atuadores de LMF para substituírem os empregados nos veículos atuais. A utilização desses atuadores de elemento de LMF reduziria o peso e o custo do automóvel produzido. Entretanto, o ramo automobilístico se encontra em estágio inicial de implementação desses atuadores, e é esperado que no futuro, com o melhor desenvolvimento de material cientifico de LMF, a utilização desses novos atuadores se torne mais frequente nas indústrias automobilísticas.

$\mathrm{Na}$ área eletrônica existe uma quantidade elevada de patentes registradas relacionadas com LMF, sendo em geral também relacionada com a produção de atuadores. Entretanto, não foi encontrada nenhum produto produzido pelas empresas analisadas (Panasonic e Konica Minolta) o que nos leva a conclusão que as empresas deste campo não estão inserindo em sua produção as ideias cientificas produzidas de LFM.

\section{Agradecimentos}

Os autores são gratos a CNPq e a FAPESP pelo suporte financeiro, ao Programa de Pós-Graduação em Ciência e Engenharia de Materiais (PPG-CEM) da Universidade Federal de São Carlos (UFSCar) e a CAPES, sob o programa BRAGECRIM em colaboração com a DFG, pelas oportunidades oferecidas que favoreceram a elaboração desse projeto de pesquisa.

\section{REFERÊNCIAS}

1 Otsuka, K.; Wayman, CME. Shape Memory Materials, Cambridge University Press, 1998.

2 Sutou, Y.; Omori, T.;Characteristics of Cu-Al-Mn-based shape memory alloys and their applications. Materials Science and Engineering: A. 2004; 378(1-2):278-282. 
3 Cava RD, Bolfarini C, Kiminami CS, Mazzer EM, Botta Filho WJ, Gargarella P, Eckert J , et al. Spray forming of Cu-11.85Al-3.2Ni-3Mn (wt\%) shape memory alloy. Journal of Alloy and Compounds. 2013; 615:602-606.

4 Mazzer EM, Kiminami CS, Bolfarini C, Cava RD, Botta Filho WJ, Gargarella P. Thermodynamic analysis of the effect of annealing on the thermal stability of a $\mathrm{Cu}-\mathrm{Al}-$ $\mathrm{Ni-Mn}$ shape memory alloy. Thermochimica Acta. 2015; 608: p. 1-6.

5 da Silva MR, Gargarella P, Gustmann T, Botta Filho WJ, Kiminami CS, Eckert J, Pauly $\mathrm{S}$, Bolfarini $\mathrm{C}$, et al. Laser surface remelting of a Cu-Al-Ni-Mn shape memory alloy. Material Science Engineering: A. 2016; 661: p. 61 - 67.

6 Mohd Jani J, Leary M. A review of shape memory alloy research, applications and opportunities. Materials \& Design. 2014; 56:1078-1113.

7 Derwent Innovations Index ®. Quick Reference Card. [acesso em 1 ago. 2014];

Disponível em:

http://www.sbu.unicamp.br/fontes-

eletronicas/images/PDF/derwentinnovationsindex_pt.pdf

$8 \quad$ Finding Technology Using Patents, An introduction; World Intellectual Property Organization.

9 The United States Patents and Trademark Office (USPTO); The USPTO: Who we are. [acesso em 1 ago. 2014]. Dísponivel em: http://www.uspto.gov/about/index.jsp

10 General, G.; Chevrolet Debuts Lightweight "Smart Material" on corvette. Motors News. 2013. 